\title{
Participant-perceived understanding and perspectives on pharmacogenomics: the Mayo Clinic RIGHT protocol (Right Drug, Right Dose, Right Time)
}

\author{
Janet E. Olson, $\mathrm{PhD}^{1}$, Carolyn R. Rohrer Vitek, MS², Elizabeth J. Bell, MPH, PhD', \\ Michaela E. McGree, BS', Debra J. Jacobson, MS', Jennifer L. St. Sauver, PhD 1,3, \\ Pedro J. Caraballo, MD ${ }^{4}$, Joan M. Griffin, $\mathrm{PhD}^{1,2}$, Veronique L. Roger, MD ${ }^{1,3,5}$ and \\ Suzette J. Bielinski, PhD ${ }^{1}$
}

Purpose: To examine predictors of understanding preemptive CYP2D6 pharmacogenomics test results and to identify key features required to improve future educational efforts of preemptive pharmacogenomics testing.

Methods: One thousand ten participants were surveyed after receiving preemptive CYP2D6 pharmacogenomics test results.

Results: Eighty-six percent $(n=869)$ of patients responded. Of the responders, $98 \%$ were white and $55 \%$ were female; $57 \%$ had 4 years or more of post-secondary education and an average age of $58.9 \pm 5.5$ years. Twenty-six percent said that they only somewhat understood their results and $7 \%$ reported they did not understand them at all. Only education predicted understanding. The most common suggestion for improvement was the use of layperson's terms when reporting results. In addition, responders suggested that results should be

\section{INTRODUCTION}

Preemptive pharmacogenomic testing is drug-gene testing performed prior to a clinical need so that the data are already available at the point of care. A recent publication outlined implementation of preemptive pharmacogenomics testing at five clinical centers and reported the types of genotype platforms used, the numbers of genes assayed, and clinical decision support used to aid prescribers. ${ }^{1}$ However, little research has been conducted to determine how best to return and explain test results to patients prior to the actual time of the prescription.

Learning how to best return and explain preemptive pharmacogenomic test results has become especially critical with the advent of national interest in tailoring all types of clinical care to each patient ("personalized medicine"). In particular, pharmacogenomics and the return of research results are two key components of the Precision Medicine Initiative (PMI) proposed by the National Institutes of Health in $2015 .^{2}$ The PMI personalized by referring to medications that they were currently using. Of those reporting imperfect drug adherence, most (91\%) reported they would be more likely to use medication as prescribed if pharmacogenomic information was used to help select the drug or dose.

Conclusion: Despite great efforts to simplify pharmacogenomic results (or because of them), approximately one-third of responders did not understand their results. Future efforts need to provide more examples and tailor results to the individual. Incorporation of pharmacogenomics is likely to improve medication adherence.

Genet Med advance online publication 5 January 2017

Key Words: CYP2D6; patient understanding; pharmacogenomics; preemptive genotyping; return of results

Cohort Program has a goal of developing a cohort of more than 1 million people in the United States to improve our knowledge of the "biological, environmental, and behavioral influences...of diseases". The current plan is to allow the PMI Cohort Program participants to access their own genomic data, including pharmacogenomic results. However, there is scarce knowledge regarding returning such results outside of the clinical setting. Traditionally, pharmacogenomics tests have been ordered by clinicians at the time that a new medication is being considered. If results were returned to patients at all, such results were interpreted by the provider in the context of the medication being considered. However, the PMI cohort members, like patients receiving preemptive pharmacogenomic results, will receive their pharmacogenomics results independent of medication prescriptions. It is currently unclear how such results should be returned to ensure that patients find the results of preemptive pharmacogenomics testing useful and easy to understand.

The Mayo Clinic's RIGHT Protocol (right drug, right dose, right time) is a study of preemptive pharmacogenomics

${ }^{1}$ Department of Health Sciences Research, Mayo Clinic, Rochester, Minnesota, USA; ${ }^{2}$ Center for Individualized Medicine, Mayo Clinic, Rochester, Minnesota, USA; ${ }^{3}$ Center for the Science of Health Care Delivery, Mayo Clinic, Rochester, Minnesota, USA; ${ }^{4}$ Department of General Internal Medicine, Mayo Clinic, Rochester, Minnesota, USA; ${ }^{5}$ Division of Cardiology, Mayo Clinic, Rochester, Minnesota, USA. Correspondence: Janet E. Olson (olsonj@mayo.edu)

Submitted 18 July 2016; accepted 16 October 2016; advance online publication 5 January 2017. doi:10.1038/gim.2016.192 
involving 1,013 patients. ${ }^{3}$ CYP2D6 genotypes were among the first clinically validated pharmacogenomic results deposited into participants' electronic health records (EHRs).

The CYP2D6 gene encodes for an important enzyme that is involved in the metabolism of xenobiotics, including up to $25 \%$ of all prescribed medications. ${ }^{4}$ However, the standard clinical genetic test report can be difficult to comprehend because it includes technical jargon and unfamiliar terms. Complicating this is the fact that the effects of various CYP2D6 variants vary depending on the form of the medication (e.g., prodrug versus active form) at the time of administration. Some medications, such as codeine, require activation by CYP2D6 before they have their intended pharmacologic effect. Patients with an "ultrarapid-metabolizer" phenotype would rapidly convert codeine to morphine and experience overdose symptoms. Other medications, such as Prozac, are administered in an active form and then deactivated by CYP2D6 prior to elimination from the body. The same patients with the ultrarapid-metabolizer phenotype would experience lack of efficacy as the medication was rapidly deactivated. Such patients require higher than normal doses to achieve therapeutic effectiveness.

To call attention to the availability of the results in EHRs and provide assistance in the interpretation of those results, we mailed a letter with a summary of results and educational materials. The aims of this study were to (i) assess the variation in the perceived understanding of results, including potential demographic differences in perceived understanding; (ii) investigate attitudes and beliefs about sharing and using CYP2D6 test results; and (iii) explore patients' ideas for improving materials used to present and interpret results.

\section{MATERIALS AND METHODS}

\section{Setting and patient selection}

The RIGHT Protocol ${ }^{3}$ was designed to test whether health-care providers can use preemptive pharmacogenomics test results to guide patient prescriptions at the point of care. We also wanted to study the effects of preemptive genotyping on the health-care utilization and outcomes of primary-care patients. Participants were selected from the Mayo Clinic Biobank ${ }^{5}$ based on age, sex, and race. ${ }^{3} \mathrm{~A}$ total of 2,000 subjects were identified; 1,013 participants provided a blood sample and informed consent and were included in the RIGHT Protocol. We conducted clinical CYP2D6 genetic testing for all participants and deposited results in the EHR. Methods for interpreting pharmacogenomic variants have been previously described. ${ }^{6}$ At the time of this study, the Mayo Clinic used the following CYP2D6 nomenclature groups: ultrarapid, extensive to ultrarapid, extensive, intermediate to extensive, intermediate, poor to intermediate, and poor. In addition, one Mayo Clinic-specific nomenclature category was used (i.e., intermediate to ultrarapid) for six patients with indeterminate copy number variations. Of the 1,013 participants, 1,010 were alive and able to participate in the current study.

\section{Study setting}

This study was approved by the Mayo Clinic Institutional Review Board. Study participants were mailed a packet of materials that included a results letter summarizing their phenotypic CYP2D6 metabolizer status. Metabolizer status was divided into two phenotypic categories: actionable and nonactionable. Each category was then further categorized into groups to describe the rate at which patients metabolize the CYP2D6 medication. For nonactionable or "normal" phenotypes, patients received a result summary saying "You process CYP2D6-related medications at a regular rate." The pharmacogenomic-based recommendation for individuals with these nonactionable CYP2D6 phenotypes (Mayo Clinic nomenclature groups: extensive, intermediate to extensive, and intermediate) is to adhere to standard drugs and/or dosages. The second category included actionable CYP2D6 phenotypes (i.e., ultrarapid, extensive to ultrarapid, intermediate to ultrarapid, poor to intermediate, and poor). At the Mayo Clinic, it is typically recommended that individuals in this category change their dose or use an alternative medication. For individuals with these phenotypes, the summary letter said "You process some medications differently." Note that genotype and phenotype methods used at the Mayo Clinic during the time of this study are not necessarily those used across the United States. For all RIGHT participants, the mailed packet also included (i) an educational brochure (see Supplementary Materials online) with one page of information about how to log onto the Mayo Clinic Patient Portal to access results, (ii) two pages of educational material about pharmacogenomics testing and CYP2D6, (iii) a nine-page survey, and (iv) a postage-paid return envelope.

\section{Data collection}

The mailed survey contained attitudinal questions about the result letter, the educational materials, participants' beliefs about personal medication use, plans for using CYP2D6 results, and the usefulness of pharmacogenomics testing in general. The questions in the survey were developed by the study investigators. Study-material questions concerning perceived understanding allowed responders to report if they understood "completely," "mostly," "somewhat," or "not at all" (Supplementary Table S1 online). Space was provided for participants to comment on things that were helpful and things that would have made the study materials more helpful. Questions about their confidence in their ability to explain their results to a friend or family member included categories of "extremely," "somewhat," "a little," and "not at all." Those who had not responded within 30 days of the initial mailing were sent a second survey. Data collection was closed 30 days after the second mailing. No incentives or follow-up telephone calls were provided.

\section{Data analyses}

To assess potential nonresponse bias, demographic and CYP2D6 phenotype data were compared for survey responders and nonresponders using a chi-square or Fisher's exact test 
for categorical variables and a $t$-test for continuous variables, as appropriate. Between-group variance was similar between compared groups. Associations between participant characteristics-including age, sex, education, CYP2D6 phenotype, number of prescription medications, marital status, self-reported health status, severity- and age-weighted sum of diseases, and perceived understanding of the CYP2D6 results and their utility-were assessed using logistic regression and summarized using odds ratios (OR) and 95\% confidence intervals (CI). For these analyses, "completely understood" and "mostly understood" were combined and compared with responses from a combined group of those who reported understanding only "somewhat" or "not at all." For attitudinal questions about encouraging others to consider undergoing pharmacogenomics testing, we used unadjusted logistic regression models.

Responses to the open-ended question "What is the one thing that would have made your result letter more helpful?" were entered into a database, coded, and analyzed for common themes by two independent reviewers (J.E.O. and M.E.M.). Discrepancies were reviewed and discussed until a consensus was reached. If a consensus could not be reached, then a third investigator (C.R.V.) determined the final code.

\section{RESULTS}

A total of 1,010 participants were alive and eligible for the survey at the time of mailing. Of these, 869 (86\%) responded to the survey (Table 1). Despite the high response rate, we observed differences between responders and nonresponders for multiple characteristics, including sex, education level, and marital status (Table 1). Responders were more likely to be female, have a higher level of education, and be married. Ninety-eight percent were white, $55 \%$ were female, and $57 \%$ had had at least 4 years of postsecondary education; the mean age was $59( \pm 5.5)$ years (Table 1). The prominence of white race and high education level among responders was consistent with overall education levels in the Mayo Clinic Biobank, the source population from which this study sample was drawn. ${ }^{5}$ Eighty-seven percent had EHR data indicating use of at least one prescription medication in 2014. More than one-third (39\%) of responders had CYP2D6 test results classified by the study team as actionable.

\section{Perceived understanding of results}

To the question "How well do you feel you understand or don't understand your CYP2D6 result?", the majority (67\%) responded that they either completely or mostly understood their results. Of concern, 33\% reported that they either only somewhat understood (26\%) or did not understand their results at all (7\%). We examined the influence of several factors on failure to understand results (Table 2). Key variables included age, sex, education, CYP2D6 phenotype, and number of prescription medications in 2014. Other covariates examined included marital status, self-reported health status, and perceived health status (Table 2). Of these, less education was associated with failure to understand results. Compared to those with a 4-year college degree or greater, those with a high school education or less or those with some higher education but not a 4-year degree were 1.6 times more likely to report understanding somewhat or not at all $(P=0.006)$.

To understand patient-perceived understanding of laboratory reports with CYP2D6 results, we asked participants to indicate their level of agreement with the following statement: "It was easy to understand my pharmacogenomic result in the Patient Portal." Of the 499 patients who said they had viewed their Patient Portal results, more than half (53\%) agreed (15\% strongly and 38\% somewhat), $12 \%$ neither agreed nor disagreed, $33 \%$ disagreed ( $21 \%$ somewhat and 12\% strongly), and $1 \%$ did not specify.

Participants were also asked to indicate their confidence level in their ability to explain their results to a friend or family member. Thirty-eight percent responded "somewhat confident." Responses, however, varied by education. Thirteen percent $(10 / 77)$ of those with a high school education or less and $23 \%(48 / 211)$ of those with a graduate or professional degree responded that they were "extremely confident." Conversely, $30 \%(23 / 77)$ of those with a high school education or less responded "not at all confident"; however, even 18\% (38/211) of those with a graduate or professional degree gave the same response.

\section{Participant attitudes regarding pharmacogenomics testing}

To determine the value placed by participants on pharmacogenomics testing of CYP2D6, we summarized responses to four questions: (i) whether they reported sharing or were planning to share their results with others, (ii) whether they would encourage others to undergo pharmacogenomics testing, (iii) how useful they believed pharmacogenomic results to be, and (iv) whether the use of pharmacogenomics would affect their medication compliance.

Of the $69 \%(N=588)$ who had shared or planned to share their CYP2D6 test results, $83 \%$ planned to share their results with a health-care provider, $60 \%$ with a spouse or partner, $30 \%$ with children, $20 \%$ with siblings, $10 \%$ with friends, $7 \%$ with parents, and $17 \%$ with a pharmacist. Some (17\%) planned to discuss or share with no one; $14 \%$ were unsure whether they would discuss or share results with anyone. Note that these categories were nonexclusive.

In general, $64 \%$ of the participants would encourage others to consider undergoing pharmacogenomics testing. The strongest predictor of who would encourage others was the reported level of understanding of the CYP2D6 result. Those who reported either completely or mostly understanding their result were more likely to encourage others to be tested than those who reported understanding their results only somewhat or not at all $(\mathrm{OR}=1.82,95 \% \mathrm{CI} 1.34-2.46)$. Other factors considered (age, sex, education, CYP2D6 phenotype, marital status, health status) were not associated with the likelihood of encouraging others to be tested.

Participant attitudes regarding the usefulness of pharmacogenomics testing are summarized in Figure 1. Responders were generally positive concerning the usefulness of 
Table 1 Baseline characteristics

\begin{tabular}{|c|c|c|c|}
\hline Characteristic & $\begin{array}{l}\text { Nonresponder } \\
\quad(N=141)\end{array}$ & $\begin{array}{l}\text { Responder } \\
(N=869)\end{array}$ & $P^{a}$ \\
\hline Male, $N(\%)$ & $78(55.3)$ & $394(45.3)$ & 0.03 \\
\hline White, $N(\%)$ & $135 / 139(97.1)$ & $842 / 860(97.9)$ & 0.53 \\
\hline Age (years), mean (SD) & $58.2(5.0)$ & $58.9(5.5)$ & 0.16 \\
\hline Age (years), $N(\%)^{b}$ & & & 0.18 \\
\hline$<55$ & $35(24.8)$ & $191(22.0)$ & \\
\hline $55-64$ & $92(65.2)$ & $540(62.1)$ & \\
\hline$\geq 65$ & $14(9.9)$ & $138(15.9)$ & \\
\hline CYP2D6 phenotype, $N(\%)$ & & & 0.03 \\
\hline Ultrarapid & $15(10.6)$ & $68(7.8)$ & \\
\hline Extensive to ultrarapid & $17(12.1)$ & $141(16.2)$ & \\
\hline Extensive & $36(25.5)$ & $169(19.4)$ & \\
\hline Intermediate to extensive & $36(25.5)$ & $161(18.5)$ & \\
\hline Intermediate & $18(12.8)$ & $200(23.0)$ & \\
\hline Poor to intermediate & $9(6.4)$ & $66(7.6)$ & \\
\hline Poor & $10(7.1)$ & $64(7.4)$ & \\
\hline CYP2D6 phenotype, $N(\%)^{c}$ & & & 0.52 \\
\hline Nonactionable & $90(63.8)$ & $530(61.0)$ & \\
\hline Actionable & $51(36.2)$ & $339(39.0)$ & \\
\hline Education, $N(\%)$ & & & 0.003 \\
\hline High school graduate or less & $22(15.6)$ & $80(9.2)$ & \\
\hline $\begin{array}{l}\text { Some college/vocational/ } \\
\text { technical/associates } \\
\text { degree including } \\
\text { community college }\end{array}$ & $63(44.7)$ & $293(33.7)$ & \\
\hline Four-year college graduate & $29(20.6)$ & $273(31.4)$ & \\
\hline $\begin{array}{l}\text { Graduate or professional } \\
\text { school }\end{array}$ & $26(18.4)$ & $218(25.1)$ & \\
\hline Unknown & $1(0.7)$ & $5(0.6)$ & \\
\hline \multicolumn{2}{|c|}{ Prescription medications in $2014, N(\%)$} & & 0.02 \\
\hline None & $32(22.7)$ & $109(12.5)$ & \\
\hline 1 to 2 & $33(23.4)$ & $207(23.8)$ & \\
\hline 3 to 5 & $32(22.7)$ & $262(30.1)$ & \\
\hline 6 to 10 & $22(15.6)$ & $177(20.4)$ & \\
\hline 11 to 20 & $17(12.1)$ & $91(10.5)$ & \\
\hline$\geq 21$ & $5(3.5)$ & $23(2.6)$ & \\
\hline Marital status, $N(\%)$ & & & 0.01 \\
\hline Single/divorced/widowed & $31(22.0)$ & $122(14.0)$ & \\
\hline Married & $110(78.0)$ & $747(86.0)$ & \\
\hline Health (self-reported) & & & - \\
\hline Excellent/very good/good & - & $816(93.9)$ & \\
\hline Fair/poor & - & $42(4.8)$ & \\
\hline Not reported & - & $11(1.3)$ & \\
\hline $\begin{array}{l}\text { Severity- and age-weighted } \\
\text { sum of diseases, mean (SD) }\end{array}$ & $2.8(2.2)$ & $2.9(2.4)$ & 0.79 \\
\hline
\end{tabular}

SD, standard deviation.

${ }^{a} C$ hi-square or Fisher's exact $P$ value presented for categorical variables and $t$-test $P$ value is presented for age and severity- and age-weighted sum of diseases. ${ }^{\text {b}}$ Age for nonresponders was calculated as the patient's age when the survey was sent out; age for responders was calculated as the patient's age when the survey was completed. Phenotypes categorized as nonactionable are extensive, intermediate to extensive, and intermediate; phenotypes categorized as actionable are ultrarapid, extensive to ultrarapid, intermediate to ultrarapid, poor to intermediate, and poor. pharmacogenomics testing. Most participants (87\%) strongly or somewhat agreed with the statement "Using my pharmacogenomic results when prescribing medications for me will improve my chances of getting a dose that is right for me." In addition, $87 \%$ strongly or somewhat agreed with the statement "Using my pharmacogenomic results when prescribing medications for me will improve my chances of getting a medication that is right for me." Participants were least likely to agree with the statement "Knowing my pharmacogenomic results will be useful for my children" (447/669, 67\%), even after the responses of those without children were removed from the analysis.

Finally, 513 (59\%) reported that they did not always follow medication instructions as directed. However, most of these participants reported that they would be either "much more likely" (67\%) or "somewhat more likely" (24\%) to take the medication as prescribed if pharmacogenomic information were used to in the selection of the medication and the dose.

\section{Participant suggestions for improving results}

We asked participants the open-ended question "What is one thing that would have made your result letter more helpful?" Three overlapping themes emerged from the analysis of responses. First, explanations of results need to be translated into plain language, preferably in person. Second, results need to be personalized to be useful for patients. Third, the layout and content of information could be simplified (Table 3).

The most common responses suggested that results be translated into plain language and emphasized the need to simplify language and to have the information delivered by someone either in person or over the phone who can explain the results. The second suggestion was that results be personalized in a way that helps patients better manage their care. Many responders requested a table or list of medications affected by CYP2D6; comments frequently included requests for matching results with current medications. The third theme included suggestions for improving the presentation of the data. One person expressed a preference for "comparative information in graph form-more visual understanding of my particular info." Another suggested personalizing results in a clearer way, asking for "information about the range of results-where do I fall in a range as an intermediate metabolizer?"

\section{DISCUSSION}

The goals of this work were to (i) determine the variation in the perceived understanding of results, (ii) investigate attitudes and beliefs about sharing and using CYP2D6 test results, and (iii) explore patients' ideas for improving materials that present and interpret results. This information is especially important as plans unfold for participants in large research initiatives, including the Precision Medicine Initiative now under way. We found that our first attempt to return CYP2D6 phenotype results to participants in our study in an understandable way was only partially successful, but it identified key elements required for improving future efforts to return pharmacogenomics results. 
Table 2 Associations with not understanding (somewhat or not at all) the CYP2D6 results returned in the results letter

\begin{tabular}{|c|c|c|c|c|}
\hline Characteristic & $\begin{array}{l}\text { Unadjusted } \\
\text { OR }(95 \% \mathrm{Cl})\end{array}$ & $P$ & $\begin{array}{l}\text { Adjusted }^{\mathrm{a}} \\
\text { OR }(95 \% \mathrm{Cl})\end{array}$ & $P$ \\
\hline Age & & 0.58 & & 0.52 \\
\hline$<55$ & Reference & & Reference & \\
\hline $55-64$ & $1.08(0.76,1.54)$ & & $1.13(0.79,1.62)$ & \\
\hline $65+$ & $1.28(0.80,2.03)$ & & $1.33(0.82,2.15)$ & \\
\hline Sex & & 0.24 & & 0.24 \\
\hline Female & Reference & & Reference & \\
\hline Male & $0.84(0.63,1.12)$ & & $0.84(0.62,1.13)$ & \\
\hline Education & & 0.003 & & 0.006 \\
\hline $\begin{array}{l}\text { Four-year college } \\
\text { graduate or } \\
\text { greater }\end{array}$ & Reference & & Reference & \\
\hline $\begin{array}{l}\text { Some higher } \\
\text { education }\end{array}$ & $1.67(1.23,2.27)$ & & $1.63(1.19,2.22)$ & \\
\hline $\begin{array}{l}\text { High school } \\
\text { graduate or less }\end{array}$ & $1.62(0.98,2.66)$ & & $1.58(0.96,2.60)$ & \\
\hline CYP2D6 phenotype & & 0.38 & & 0.48 \\
\hline
\end{tabular}

$\begin{array}{lc}\text { Nonactionable } & \text { Reference } \\ \text { Actionable } & 1.14(0.85,1.52)\end{array}$

\begin{tabular}{|c|c|c|c|c|}
\hline \multicolumn{2}{|c|}{ Prescription medications in 2014} & 0.07 & & 0.13 \\
\hline None & Reference & & Reference & \\
\hline 1 to 2 & $1.45(0.85,2.47)$ & & $1.34(0.78,2.31)$ & \\
\hline 3 to 5 & $1.77(1.06,2.96)$ & & $1.61(0.96,2.71)$ & \\
\hline 6 to 10 & $2.15(1.25,3.68)$ & & $1.97(1.14,3.41)$ & \\
\hline 11 to 20 & $2.00(1.08,3.71)$ & & $1.74(0.92,3.27)$ & \\
\hline$\geq 21$ & $1.05(0.35,3.15)$ & & $0.82(0.27,2.53)$ & \\
\hline \multicolumn{2}{|l|}{ Marital status } & 0.60 & & 0.54 \\
\hline Married & Reference & & Reference & \\
\hline $\begin{array}{l}\text { Single/divorced/ } \\
\text { widowed }\end{array}$ & $0.89(0.59,1.36)$ & & $0.88(0.57,1.34)$ & \\
\hline \multicolumn{2}{|c|}{ Health (self-reported) } & 0.22 & & 0.36 \\
\hline $\begin{array}{l}\text { Excellent/very } \\
\text { good/good }\end{array}$ & Reference & & Reference & \\
\hline Fair/poor & $1.49(0.79,2.82)$ & & $1.36(0.71,2.60)$ & \\
\hline $\begin{array}{l}\text { Severity- and } \\
\text { age-weighted } \\
\text { sum of diseases }\end{array}$ & $1.03(0.97,1.09)$ & 0.28 & $1.02(0.96,1.09)$ & 0.53 \\
\hline
\end{tabular}

Due to missing data, the model $N$ ranged from 847 to 862 .

$\mathrm{Cl}$, confidence interval; OR, odds ratio.

aAge was adjusted for sex and education, sex was adjusted for age and education, and education was adjusted for age and sex; all others were adjusted for age, sex, and education. bPhenotypes categorized as nonactionable are extensive, intermediate to extensive, and intermediate; phenotypes categorized as actionable are ultrarapid, extensive to ultrarapid, intermediate to ultrarapid, poor to intermediate, and poor.

Most participants were enthusiastic about pharmacogenomics testing after receiving their results. Most had shared or planned to share their results with someone, would encourage others to undergo testing, and thought that the results would be useful for either their own health care or for their children.

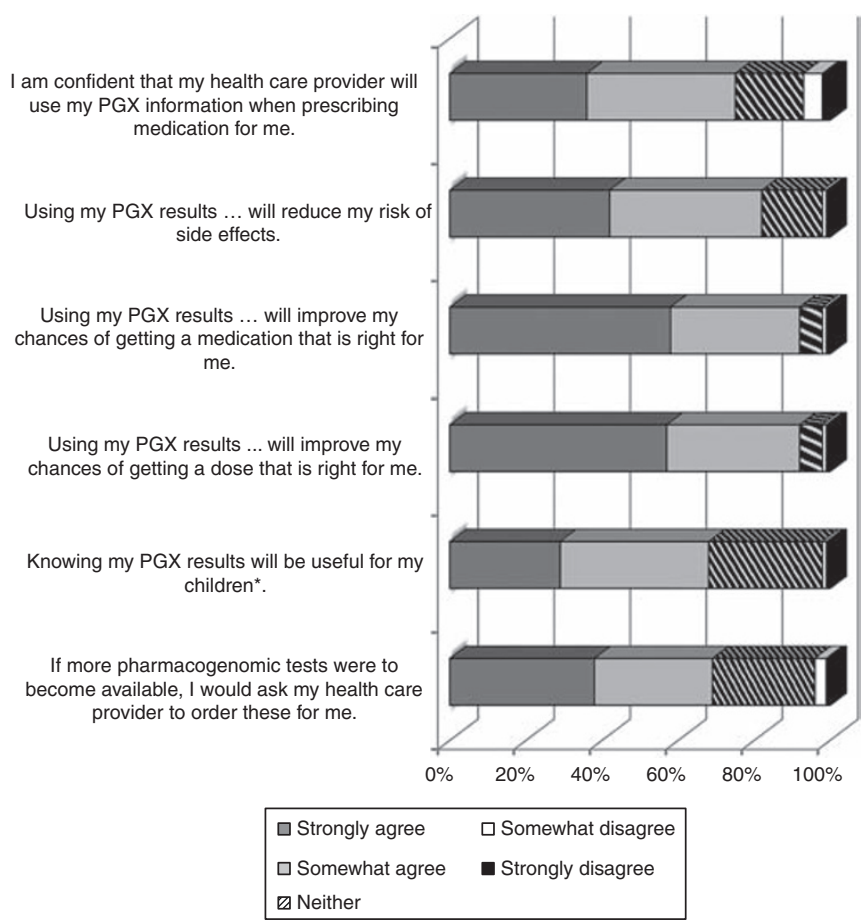

Figure 1 Participant attitudes regarding the usefulness of pharmacogenomics (PGX) testing. Patients were provided the following instructions for the statements shown: "Please indicate your agreement or disagreement with the following statements." *Among the 669 to whom it applies ("Does not apply" responses were not included in the results presented in the figure).

The results show that significant work is still needed to provide patient-centered results that meet patient needs. Providing clear and concise results while trying to balance the limitations of current science, the complexity of test results, and patient needs will continue to be a challenge as national efforts move forward. Many participants had difficulty understanding their results; those with less education were more likely to report problems with interpretation. Many participants also recommended that we improve our explanations using nontechnical language. Avoiding the standard scientific categories used by the clinical laboratory, such as "intermediate to ultrarapid metabolizer," could significantly improve interpretation. Although these are standard names for the CYP2D6 phenotypes, the actual effect of such phenotypes on a patient's response to a specific medication is difficult to interpret.

Many patients also commented that they wanted more information about which medications are affected by CYP2D6; some requested lists of medications to avoid or use with caution. More broadly, a theme that consistently emerged from the comments was that patients really wanted to know what the results meant for them personally. We tried to keep our results report simple and did not discuss all the possible ways that CYP2D6 might affect the metabolism of various medications because the impact of CYP2D6 phenotype on the response to a particular medication varies depending on whether the medication is delivered in an active state or needs to be activated to achieve 


\begin{tabular}{|c|c|c|c|}
\hline Primary theme & Subtheme & Count & Sample responses \\
\hline \multirow[t]{6}{*}{$\begin{array}{l}\text { Plain language } \\
\text { from clinician }\end{array}$} & \multirow[t]{3}{*}{ Simplify language } & \multirow[t]{3}{*}{297} & $\begin{array}{l}\text { Use layman's terms. The term "intermediate" was not explained well as being } \\
\text { "typical" or "normal." I showed this to my Mayo doctor; he said ignore it. }\end{array}$ \\
\hline & & & $\begin{array}{l}\text { The letter still had a lot of medical detail that was difficult to understand for } \\
\text { someone without a medical background }\end{array}$ \\
\hline & & & Name all genes. Not CYP2D6—call it CARL. That, I can remember. \\
\hline & \multirow{3}{*}{$\begin{array}{l}\text { Clinical assistance } \\
\text { needed }\end{array}$} & \multirow{3}{*}{14} & If my doctor or nurse had gone over the results, it would be more helpful \\
\hline & & & If the letter would have included "talk to your provider if you take... medications" \\
\hline & & & I feel this information is for the doctor and not sure I need to know this \\
\hline \multirow[t]{2}{*}{ Personalize results } & $\begin{array}{l}\text { Information for personal } \\
\text { health }\end{array}$ & 59 & Really, what does it mean for the drugs I take? \\
\hline & Medication information & 93 & How are my current meds being metabolized? \\
\hline \multirow{4}{*}{$\begin{array}{l}\text { Suggestions for } \\
\text { improvements }\end{array}$} & \multirow{2}{*}{$\begin{array}{l}\text { Electronic health record } \\
\text { suggestions }\end{array}$} & \multirow[t]{2}{*}{9} & To explain each test when clicking on it in the online results \\
\hline & & & $\begin{array}{l}\text { The letter is OK. The online comments are very involved and difficult to } \\
\text { understand. }\end{array}$ \\
\hline & \multirow[t]{2}{*}{ Suggestion on layout } & \multirow[t]{2}{*}{62} & A small graph showing where my results were as to "normal" \\
\hline & & & $\begin{array}{l}\text { Put test results in tabular form, i.e., Gene CYP2D6 Result normal } \\
\text { (extensive metabolizer). This would be particularly helpful if more than } \\
\text { one result is available. }\end{array}$ \\
\hline
\end{tabular}

its desired therapeutic benefit. ${ }^{4}$ Our decision not to present the differential activity on various medications may have inadvertently caused confusion for some participants. Interpreting CYP2D6 phenotypes in lay language and saying which drugs are likely to be affected by the phenotype could significantly improve patient satisfaction with return of results.

Finally, we found that more than half of our participants reported that they did not take their medications routinely or as directed. Medication adherence varies depending on type of condition and medication but has been reported to range from 17 to $80 \%{ }^{7}$ Therefore, our study population seemed to be typi$\mathrm{cal}$ in their medication use behavior. However, the vast majority of those who were nonadherent to their medication regimens indicated that if pharmacogenomic information were used to tailor their prescription, they would be much more likely to take their medication. Lack of medication adherence has also been strongly associated with increases in health-care utilization and cost. ${ }^{8}$ Our results suggest that, at least for a subset of the population, pharmacogenomic information or more precise tailoring of prescription medications could improve medication adherence.

Another interesting result pertained to respondent confidence that their health-care provider will use pharmacogenomic information when prescribing medication for them in the future. Unlike other questions about the value of pharmacogenomics testing, which showed very little disagreement among respondents, $7 \%$ indicated that they disagreed either strongly (5\%) or somewhat (2\%) with the statement that they were confident that their provider would use pharmacogenomic information when prescribing their medications. We found this disconnect to be of interest and plan to explore this further in future studies of patient and physician attitudes toward pharmacogenomics. To date, we have only been able to explore this preliminarily. ${ }^{9}$

Limitations of our study include the potential lack of generalizability. Our study population was highly motivated and enthusiastic about the potential for implementing pharmacogenomics in routine patient care. In addition, they were mostly white, highly educated, and in very good health. Populations with different characteristics may be less interested in pharmacogenomics testing; our results indicate that persons with less education were also less likely to understand study results. However, many people in our study population still had difficulty understanding what their pharmacogenomics results meant for their personal health. Therefore, future studies involving the return of preemptive test results will need to ensure that patient materials related to the delivery of pharmacogenomics results are tested and validated in multiple populations, with multiple education levels, to ensure that the results are simple and easy to comprehend in all populations. Another limitation is that we were unable to verify that patients had actually viewed their results in the Mayo Clinic Patient Portal. In addition, we were not able to ask specific questions about their knowledge of their results. Future research in this area is warranted.

In summary, we found that participants in a study of preemptive pharmacogenomics testing were enthusiastic about 
the potential benefits of such testing but felt that it was difficult to interpret results and that the personal impact of such test results was unclear. Future studies focused on ensuring that pharmacogenomic results are clear and easy to interpret in multiple populations will be essential for all initiatives focused on the delivery of personalized medicine.

\section{SUPPLEMENTARY MATERIAL}

Supplementary material is linked to the online version of the paper at http://www.nature.com/gim

\section{ACKNOWLEDGMENTS}

This study was funded as part of the NHGRI-supported eMERGE (Electronic Records and Genomics) Network (HG04599 and HG006379). Additional support was provided by the Mayo Center for Individualized Medicine and the Mayo Clinic Robert D. and Patricia E. Kern Center for the Science of Health Care Delivery.

\section{DISCLOSURE}

The authors declare no conflict of interest.

\section{REFERENCES}

1. Dunnenberger HM, Crews KR, Hoffman JM, et al. Preemptive clinical pharmacogenetics implementation: current programs in five US medical centers. Annu Rev Pharmacol Toxicol 2015;55:89-106.

2. Precision Medicine Initiative (PMI) Working Group Report to the Advisory Committee to the Director, NIH. The Precision Medicine Initiative Cohort Program—Building a Research Foundation for 21st Century Medicine. 2015 http://www.nih.gov/sites/default/files/research-training/initiatives/pmi/pmiworking-group-report-20150917-2.pdf. Accessed 17 March 2016.

3. Bielinski SJ, Olson JE, Pathak J, et al. Preemptive genotyping for personalized medicine: design of the right drug, right dose, right time-using genomic data to individualize treatment protocol. Mayo Clin Proc 2014;89:25-33.

4. Owen RP, Sangkuhl K, Klein TE, Altman RB. Cytochrome P450 2D6. Pharmacogenet Genomics 2009;19:559-562.

5. Olson JE, Ryu E, Johnson KJ, et al. The Mayo Clinic Biobank: a building block for individualized medicine. Mayo Clin Proc 2013;88:952-962.

6. Ji Y, Skierka JM, Blommel JH, et al. Preemptive pharmacogenomic testing for precision medicine: a comprehensive analysis of five actionable pharmacogenomic genes using next-generation DNA sequencing and a customized CYP2D6 genotyping cascade. J Mol Diagn 2016;18:438-445.

7. Krueger KP, Berger BA, Felkey B. Medication adherence and persistence: $\mathrm{a}$ comprehensive review. Adv Ther 2005;22:313-356.

8. luga AO, McGuire MJ. Adherence and health care costs. Risk Manag Healthc Policy 2014;7:35-44.

9. St Sauver JL, Bielinski SJ, Olson JE, et al. Integrating pharmacogenomics into clinical practice: promise vs reality. Am J Med 2016;129:1093-1099.e1. 